\title{
Effectiveness and safety of toripalimab, camrelizumab, and sintilimab in a real-world cohort of hepatitis B virus associated hepatocellular carcinoma patients
}

\author{
Jinzhang Chen ${ }^{1,2 \#}$, Xiaoyun $\mathrm{Hu}^{1 \#}$, Qi Li ${ }^{1,2}$, Wencong Dai ${ }^{1}$, Xiao Cheng ${ }^{1}$, Wei Huang ${ }^{3}$, Wenxuan Yu ${ }^{1}$, \\ Mian Chen ${ }^{4}$, Yabing Guo ${ }^{1}$, Guosheng Yuan ${ }^{1}$ \\ ${ }^{1}$ Department of Infectious Diseases and Hepatology Unit, ${ }^{2}$ Department of Oncology, Nanfang Hospital, Southern Medical University, Guangzhou, \\ China; ${ }^{3}$ Department of Oncology, ShunDe Hospital, Southern Medical University, Guangzhou, China; ${ }^{4}$ Transplant Immunology Laboratory, \\ Churchill Hospital, Oxford University Hospitals NHS Foundation Trust, Old Road, Headington, Oxford, UK \\ Contributions: (I) Conception and design: G Yuan, Y Guo, X Hu, M Chen, J Chen; (II) Administrative support: J Chen, Y Guo; (III) Provision of \\ study materials or patients: X Hu, Q Li, W Dai, X Cheng, Y Guo, J Chen, W Huang, W Yu; (IV) Collection and assembly of data: G Yuan, X Hu; \\ (V) Data analysis and interpretation: J Chen, G Yuan, Y Guo, X Hu, M Chen, J Chen; (VI) Manuscript writing: All authors; (VII) Final approval of \\ manuscript: All authors. \\ "These authors contributed equally to this work. \\ Correspondence to: Guosheng Yuan, PhD; Yabing Guo, MD. Department of Infectious Diseases and Hepatology, Nanfang Hospital, Southern Medical \\ University, 1838 North Guangzhou Ave., Guangzhou, China. Email: guoshengyuan1991@163.com; gyabing@126.com.
}

Background: The clinical significance of programmed cell death protein-1 (PD-1)-targeted immunotherapy in Chinese patients is understudied. We thus aimed to evaluate the safety and efficacy of PD-1 inhibitors with toripalimab, camrelizumab or sintilimab for Chinese hepatocellular carcinoma (HCC) patients in a real-life cohort.

Methods: We analysed hepatitis B virus (HBV)-associated HCC patients treated with toripalimab, camrelizumab, or sintilimab in a retrospective single-center cohort from November 2018 to June 2020. Efficacy was evaluated with objective response rate (ORR), disease control rate (DCR), progression-free survival (PFS), time to tumor progression (TTP), and overall survival (OS). Safety data were also recorded.

Results: Seventy patients were finally included in the analysis: 23 were treated with toripalimab, 33 with camrelizumab, and 14 with sintilimab. The mean duration of follow-up was 44.7 (95\% CI: 39.9-49.6) weeks and the mean cycles of PD-1 at cutoff were $8.3 \pm 8.0$ for all patients. The ORR and DCR for the whole cohort were $30 \%$ and $72.9 \%$, respectively. Overall, 25 (35.7\%) patients had radiological disease progression and $10(14.3 \%)$ patients died during follow-up. Median PFS, median TTP, and median OS had not yet been reached. Most frequent drug-related adverse events (AEs) were rash (27.1\%), hypertension (18.6\%), fatigue (17.1\%), diarrhea (17.1\%), paresthesia (15.7\%), and nausea $(15.7 \%)$.

Conclusions: Our findings suggest that (I) PD-1 targeted immunotherapy with toripalimab, camrelizumab, or sintilimab yielded a promising outcome in Chinese HBV patients with HCC and that (II) immunotherapy was well tolerated generally and had manageable side effects. This approach thus warrants further popularization and application in clinical practice.

Keywords: Hepatocellular carcinoma (HCC); programmed death receptor-1; anti-programmed cell death protein-1 antibody (anti-PD-1 antibody); toripalimab; camrelizumab; sintilimab

Submitted Aug 19, 2020. Accepted for publication Sep 14, 2020.

doi: $10.21037 /$ atm-20-6063

View this article at: http://dx.doi.org/10.21037/atm-20-6063

(c) Annals of Translational Medicine. All rights reserved. 


\section{Introduction}

Hepatocellular carcinoma (HCC) is one of the leading causes of cancer-related death worldwide, particularly in China, where HCC cases alone account for more than half of the estimated global total (1-4). The overall survival (OS) of HCC varies considerably across the world, and Taiwan and Japan, where comprehensive surveillance programs provide early detection and curative therapy, have the best clinical outcomes. The outcomes in China, along with those in North America and Europe, however, are not as good. In these countries, over $60 \%$ of patients present to the hospitals with intermediate-to-advanced stage HCC (5). Unfortunately, for most of these late-stage patients, only systemic and/or palliative treatment can be offered. The average survival time of these patients is usually less than 1 year $(6-10)$.

Sorafenib is a multi-tyrosine kinase inhibitor (TKI) that inhibits proteins involved in angiogenesis and proliferation pathways implicated in the pathogenesis of HCC, such as vascular endothelial growth factor receptors (VEGFR), Raf-1, and B-Raf (11). This drug was approved for advanced HCC treatment in 2007 following the phase III SHARP trial (12). It has proven to be the only effective first-line systemic treatment option available for over a decade. It was not until recently that a few other TKIs such as lenvatinib (13), regorafenib (14), and Cabozantinib (15) have been approved for first- and second-line HCC treatment. Although the use of TKIs has significant OS benefits over placebo, its effects are not easily sustained. Many of the treatment regimens are limited by primary and secondary resistance to the drugs, or fail due to severe treatment-related adverse events (TRAE) forcing the discontinuation of the therapy (11).

Immune checkpoint blocker therapy has demonstrated encouraging efficacy in multiple cancer types, particularly melanoma and lung cancers. A rapidly growing list of blocking antibodies to immune checkpoints has been approved for cancer treatment (16). HCC, now generally considered as an immunogenic tumor, may also be a good candidate for such therapy (17). Programmed cell death protein-1 (PD-1) is a transmembrane molecule found to be expressed on the surface of $T$ and $B$ lymphocytes, natural killer $\mathrm{T}$ cells, activated monocytes, and dendritic cells. The PD-1 molecule negatively regulates $\mathrm{T}$ cell antigen receptor (TCR) signaling by interacting with specific ligands, namely PD-L1 and PD-L2 (18-20). Aberrantly upregulated PDL1 expression has been found on many tumor cells, which is likely to result in tumor-induced immune suppression via PD-1 signaling, and in turn, favors the growth and expansion of tumor cells. Overexpression of PD-L1 has been demonstrated to be significantly associated with tumor aggression and postoperative recurrence in HCC $(21,22)$. Disrupting the interaction between PD-1 and its ligands using monoclonal antibody $(\mathrm{mAb})$ prevents the activation of its downstream signaling pathways, and restores the host antitumor immunity (23). Nivolumab and pembrolizumab are potent $\mathrm{mAbs}$ that specifically target PD-1. CheckMate-040 (NCT01658878), a phase I study of nivolumab-treated sorafenib-intolerant/refractory advanced HCC patients resulted in a $20 \%$ objective response rate (ORR) and a promising 1 year OS rate of $62 \%$ (24). Similarly in an open-label, non-randomized phase II study, KEYNOTE-224 (NCT02702401), pembrolizumab treatment had demonstrated an ORR of $16.3 \%$ and a 6-month OS rate of 77.9\% (25). Most other PD-1 inhibitor trials for advanced HCC are still at their patient recruitment or preliminary analysis stages; however, these findings were supported by an Austro-German international multicenter real-world study of 65 patients. In this study, the ORR of nivolumab ( $\mathrm{n}=34)$ was $15 \%$ while that of pembrolizumab $(n=31)$ was $10 \%$, resulting in an overall ORR of $12 \%$ for this retrospective cohort treated with PD-1 inhibitor therapy (26).

Three PD-1 mAbs have been developed and approved in China since 2016: two humanized PD-1 antibodies, including toripalimab (JS-001, Shanghai Junshi Bioscience Co. Ltd.) (27) and camrelizumab (SHR-1210, Jiangsu Hengrui Medicine Co. Ltd.) (28); and sintilimab, a fully human PD-1 mAb (IBI308, Innovent Biologics and Eli Lilly and Company) (29). Although these three drugs demonstrated efficacy in several cancer types, only camrelizumab has thus far been subjected to trial study (NCT02989922) in HCC patients, yielding an ORR of $13.8 \%$ and a 6 -month OS rate of $74.7 \%$ in a study of 217 patients (28). This ORR is comparable to the typical $10-20 \%$ observed in other PD-1 therapy trials $(24,25,28,30)$. However, being domestically developed, the most notable advantage of these drugs to Chinese patients is the significantly lower cost (approx. $\$ 17.5 \mathrm{k}$, $\$ 17.0 \mathrm{k}$, and $\$ 13.9 \mathrm{k}$ USD per year respectively), being a fraction of that of nivolumab (approx. \$63 k USD per year) and pembrolizumab (approx. $\$ 87 \mathrm{k}$ USD per year). It is important to note that China's large population of HCC patients consists of members from vastly different 
socioeconomic backgrounds, and that the overall cost of a therapy is an important determinant in patients' access to the appropriate treatments.

In China, more than $80 \%$ of HCC patients have a history of chronic hepatitis $\mathrm{B}$ virus (HBV) infection at the time of diagnosis. Persistent infection with HBV not only leads to chronic liver inflammation and drives the progression to HCC (31), it has also been shown to demonstrate many immunosuppressive properties. For example, hepatitis B core antigen ( $\mathrm{HBcAg}$ ) is capable of inducing in vivo secretion of interleukin-10 (IL-10), a potent immunosuppressive cytokine (32). We thus aimed to evaluate the safety and efficacy of these PD-1 inhibitor drugs in a real-world treatment cohort of $\mathrm{HBV}$-associated HCC patients in China. To our knowledge, this is the first report of its kind, in that it reflects the treatment reality in HCC outside of clinical trial programs. We hope to share our experience with developing countries where the majority of HCC cases are also associated with chronic $\mathrm{HBV}$ infection (3). We present the following article in accordance with the STROBE reporting checklist (available at http://dx.doi.org/10.21037/atm-20-6063).

\section{Methods}

\section{Study design and patients}

This is a single-center retrospective study of patients who received PD-1 inhibitor monotherapy with toripalimab, camrelizumab, or sintilimab, or a combination therapy with other modes of cancer treatments. Patients with chronic HBV infection and radiologically or histologically diagnosed HCC were eligible. The approval for this retrospective study was obtained from the ethical committee of Nanfang Hospital, Southern Medical University (NFEC201903-Y1-01). Written consent was obtained from patients prior to their enrolment into this study. All procedures performed in this study involving human participants were in accordance with the Declaration of Helsinki (as revised in 2013).

\section{Dosage PD-1 inhibitor therapy}

Toripalimab was given intravenously at $3 \mathrm{mg} / \mathrm{kg}$ body weight or at a fixed dose of $240 \mathrm{mg}$ every 2 weeks. Camrelizumab was given at a fixed dose of $200 \mathrm{mg}$ every $2-3$ weeks intravenously. Sintilimab was given at a fixed dose of $200 \mathrm{mg}$ every 3 weeks intravenously.

\section{Assessments}

Clinical and laboratory data were collected from all patients prior to $\mathrm{PD}-1$ inhibitor therapy. Clinical data included age, gender, Barcelona Clinic Liver Cancer (BCLC) stage, Eastern Cooperative Oncology Group (ECOG) performance, and Child-Pugh score. In addition, imaging data were collected based on abdominal computed tomography (CT) and/or magnetic resonance imaging (MRI), and included tumor size, number, vascular invasion, and extrahepatic metastasis. Laboratory data included alpha-fetoprotein (AFP), alanine aminotransferase (ALT), aspartate aminotransferase (AST), albumin, total bilirubin (TBIL), hepatitis B surface antigen (HBsAg), and prothrombin time (PT).

The patients underwent CT or MRI at baseline, 612 weeks after treatment initiation, and about every 3 months thereafter. Efficacy of treatment was measured by OS (defined as the interval between the first dose of PD-1 administration until death or the last follow-up), time to tumor progression (TTP) (defined as the time from first checkpoint inhibitor administration until the date that tumor progression was confirmed radiologically), and progression-free survival (PFS) (defined as the time from first checkpoint inhibitor administration until radiological disease progression or death, whichever came first). TRAEs were recorded at every visit according to the US National Cancer Institute (NCI) Common Terminology Criteria for Adverse Events (CTCAE v. 4.03) (33).

Tumor responses were evaluated according to the modified response evaluation criteria in solid tumor (mRECIST) (34) and included the following classifications: (I) complete response (CR), disappearance of target lesions according to all enhanced imaging in the arterial phase; (II) partial response (PR), the total reduction of the diameter of the target lesions (enhanced arterial phase) by $\geq 30 \%$; (III) stable disease (SD), the diameter of the target lesion not reduced to that in $\mathrm{PR}$ and not increased to that in progressive disease (PD); (IV) $\mathrm{PD}$, the diameter of the target lesion increased by at least $20 \%$ compared with the 
baseline value or the appearance of new lesions according to enhanced imaging in the arterial phase.

\section{Statistical analysis}

Statistical analysis was performed in SPSS Statistics for Windows (version 20.0, SPSS Inc., Chicago, IL, USA). Data are expressed as counts and percentages for categorical variables such as those in baseline characteristics, radiological tumor response, and side effects. Kaplan-Meier survival curves were plotted for all groups. All statistical analyses were based on two-tailed hypothesis tests with a significance level of $\mathrm{P}<0.05$. Comparison between three PD-1 inhibitor groups were not performed due to the low sample size apparent after the cohort was subdivided.

\section{Results}

\section{Patients}

Between November 2018 and June 2020, a total of 146 HCC patients received PD-1-targeted immunotherapy in our hospital (Figure 1). The data collection cutoff time was June 30, 2020. Seventy-six patients were excluded because of incomplete data $(\mathrm{n}=63)$ or follow-up shorter than 6 weeks $(\mathrm{n}=13)$. The remaining 70 patients were divided into three treatment groups based on the antibody used: toripalimab $(n=23)$, camrelizumab $(n=33)$, and sintilimab $(n=14)$. The demographic and laboratory characteristics at baseline are shown in Table 1. The mean duration of followup was 44.7 (95\% CI: 39.9-49.6) weeks for all patients, 59.0 (95\% CI: 48.8-69.2) weeks for the toripalimab group, 37.0 (95\% CI: 36.2-37.8) for the camrelizumab group, and 49.0 (95\% CI: $36.4-61.6$ ) for the sintilimab group. The mean number of cycles of PD-1 therapy at cutoff were $8.3 \pm 8.0$ for all patients, and $7.4 \pm 2.9,7.0 \pm 2.7$, and $12.6 \pm 16.9$ for the toripalimab, camrelizumab, and sintilimab groups, respectively.

\section{Efficacy}

Seventy patients who had at least one follow-up imaging result were included in the assessment of tumor responses (Table 2). In the toripalimab group $(\mathrm{n}=23)$, no patient had complete response (CR), 5 patients achieved PR, and 10 patients had SD, resulting in an ORR of $21.7 \%$ and a disease control rate (DCR) of $56.5 \%$. In the camrelizumab-treated patients ( $\mathrm{n}=33$ ), the ORR and DCR were $36.4 \%$ and $81.8 \%$, respectively, with 2, 10, and 15 patients experiencing CR, $\mathrm{PR}$, and SD, respectively. In the sintilimab group $(\mathrm{n}=14)$, 1 and 3 participants achieved CR and PR, respectively, with 7 patients experiencing SD and 3 experiencing PD. The ORR was $28.6 \%$, and the DCR was $78.6 \%$ for this treatment group. The overall ORR and DCR of the entire cohort were $30.0 \%$ and $72.9 \%$, respectively. However, due to the heterogeneous nature of patients and these therapies, the amount of data generated from this small cohort was insufficient for providing any statistical conclusions.

The median PFS of the entire cohort, and that of the camrelizumab and sintilimab groups, had not yet been reached (Figures 2,3, Table 2). The median PFS of the toripalimab group was 48 weeks (95\% CI: not reached) (Figure 3, Table 2). The PFS probability at 52 weeks was $57.0 \%$ (95\% CI: $42.5-71.5 \%$ ) for the whole population (Table 2), and 46.4\% (95\% CI: 25.2-67.6\%), 60.7\% (95\% CI: $33.5-87.9 \%$ ), and $58.9 \%$ (95\% CI: $29.7-88.1 \%$ ) for the toripalimab, camrelizumab. and sintilimab groups (Table 2). Overall, 25 (35.7\%) patients had radiological disease progression during follow-up. TTP was equal to PFS (Figures 4,5, Table 2). Ten patients (10/70, 14.3\%) died during follow-up. The median OS of the entire cohort, and the toripalimab, camrelizumab, and sintilimab groups had not yet been reached (Figures 6,7, Table 2). The OS probability at 52 weeks was $84.1 \%$ (95\% CI: $74.9-93.3 \%$ ) for the entire cohort (Table 2), and 82.6\% (95\% CI: 67.198.1\%), $83.1 \%$ (95\% CI: $66.2-100.0 \%$ ), and $83.6 \%$ (95\% CI: $62.4-104.8 \%)$ for the toripalimab, camrelizumab, and sintilimab groups (Table 2). The OS probability at 26 weeks (6 months) was 95.7\% (95\% CI: 87.3-104.1\%), 93.9\% (95\% CI: $85.7-102.1 \%$ ), and $92.9 \%$ (95\% CI: $79.4-106.4 \%)$ for the toripalimab, camrelizumab, and sintilimab groups.

\section{Safety}

All recorded TRAEs are shown in Table 3. Thirty patients (30/70, 42.9\%) experienced at least one adverse event (AE). Overall, the most frequent AEs were rash (19/70, 27.1\%), hypertension $(13 / 70,18.6 \%)$, fatigue $(12 / 70,17.1 \%)$, diarrhea $(12 / 70,17.1 \%)$, paresthesia $(11 / 70,15.7 \%)$, and nausea $(11 / 70,15.7 \%)$. One patient died from myocarditis, which was probably related to camrelizumab as it erupted after one cycle of treatment. This patient did not inform his doctor in the early stage of myocarditis, resulting in delayed treatment, and was in an incurable state. A dose 


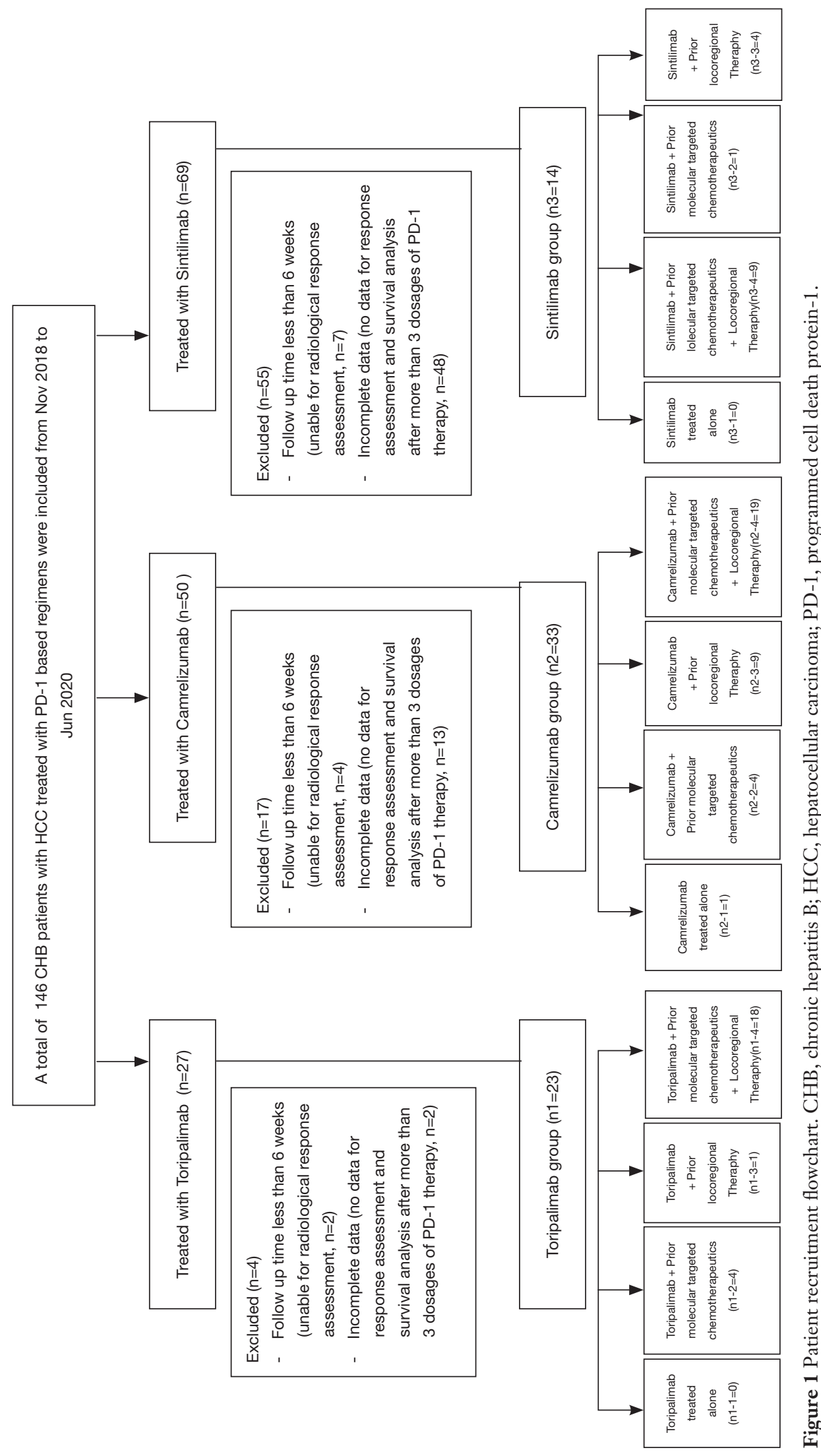


Table 1 Baseline patient characteristics

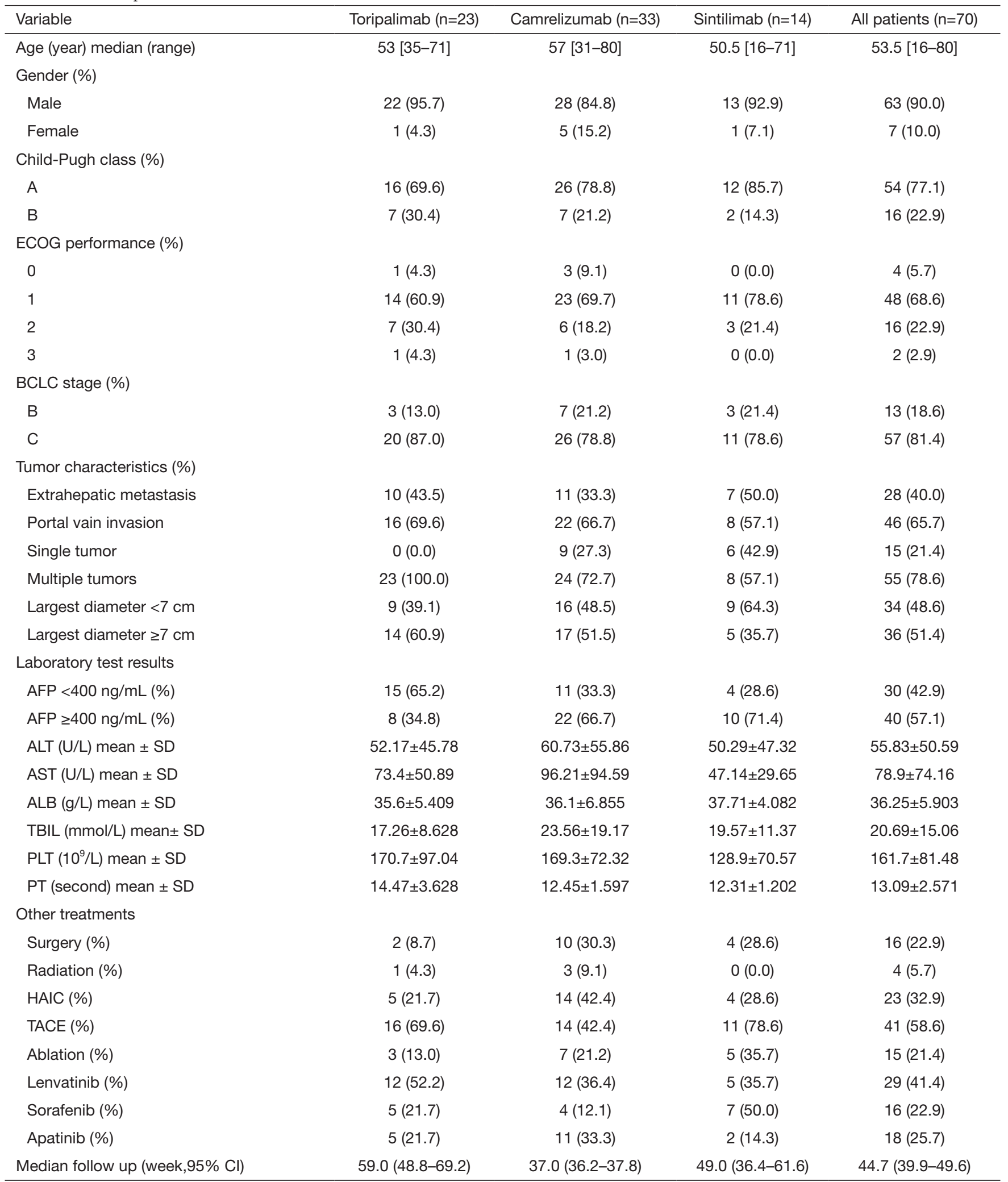

BCLC, Barcelona-Clinic Liver Cancer; ECOG, Eastern Cooperative Oncology Group; ALT, alanine aminotransferase; AST, aspartate aminotransferase; TBIL, total bilirubin; PLT, platelet count; ALB albumin; AFP, $\alpha$-fetoprotein; HAIC, hepatic artery infusion chemotherapy; TACE, transarterial chemoembolization. 
Table 2 Tumor responses and survival

\begin{tabular}{|c|c|c|c|c|}
\hline Outcome & Toripalimab $(\mathrm{n}=23)$ & Camrelizumab (n=33) & Sintilimab (n=14) & All patients $(n=70)$ \\
\hline \multicolumn{5}{|l|}{ Tumor response } \\
\hline Complete response (CR) & $0(0.0 \%)$ & $2(6.1 \%)$ & $1(7.1 \%)$ & $3(4.3 \%)$ \\
\hline Partial response (PR) & $5(21.7 \%)$ & $10(30.3 \%)$ & $3(21.4 \%)$ & $18(25.7 \%)$ \\
\hline Stable disease (SD) & 8 (34.8\%) & $15(45.5 \%)$ & 7 (50.0\%) & $30(42.9 \%)$ \\
\hline ORR (CR + PR) & $5(21.7 \%)$ & $12(36.4 \%)$ & $4(28.6 \%)$ & $21(30.0 \%)$ \\
\hline $\mathrm{DCR}(\mathrm{CR}+\mathrm{PR}+\mathrm{SD})$ & $13(56.5 \%)$ & 27 (81.8\%) & $11(78.6 \%)$ & $51(72.9 \%)$ \\
\hline \multicolumn{5}{|l|}{ Survival (weeks) } \\
\hline PFS median (95\% Cl) & 48.0 (NR-NR) & NR & NR & NR \\
\hline OS median $(95 \% \mathrm{Cl})$ & NR & NR & NR & NR \\
\hline 52 weeks OS\% $(95 \% \mathrm{Cl})$ & $82.6(67.1-98.1)$ & $83.1(66.2-100.0)$ & $83.6(62.4-104.8)$ & 84.1 (74.9-93.3) \\
\hline
\end{tabular}

$\mathrm{CR}$, complete response; PR, partial response; SD, stable disease; PD, progressive disease; ORR, objective response rate; DCR, disease control rate; PFS, progression-free survival; TTP, time to tumor progression; OS, overall survival; NR, not reached

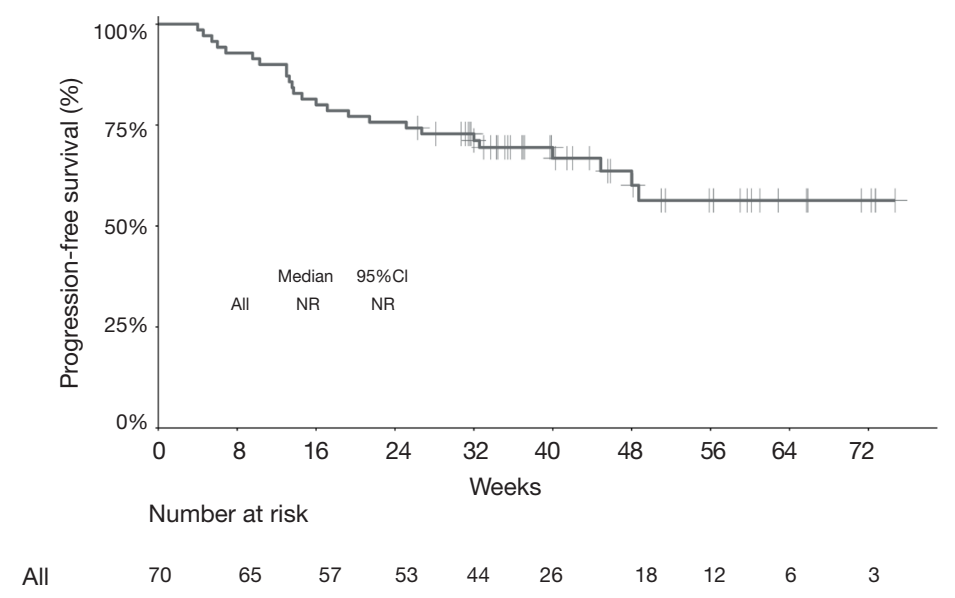

Figure 2 Kaplan-Meier curve showing progression-free survival for the entire cohort of patients treated with programmed cell death protein-1 (PD-1)-targeted immunotherapy. NR, not reached. CI, confidence interval. 


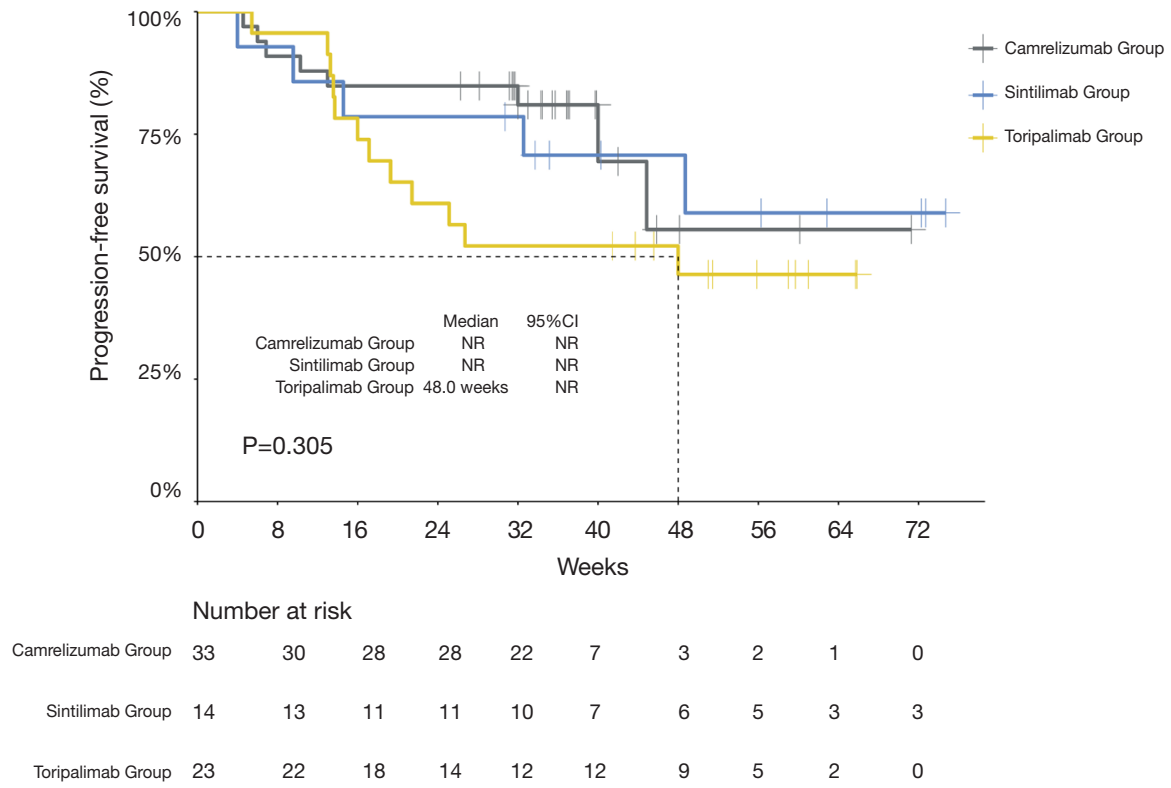

Figure 3 Kaplan-Meier curves showing progression-free survival for toripalimab-, camrelizumab-, and sintilimab-treated patients. NR, not reached; CI, confidence interval.

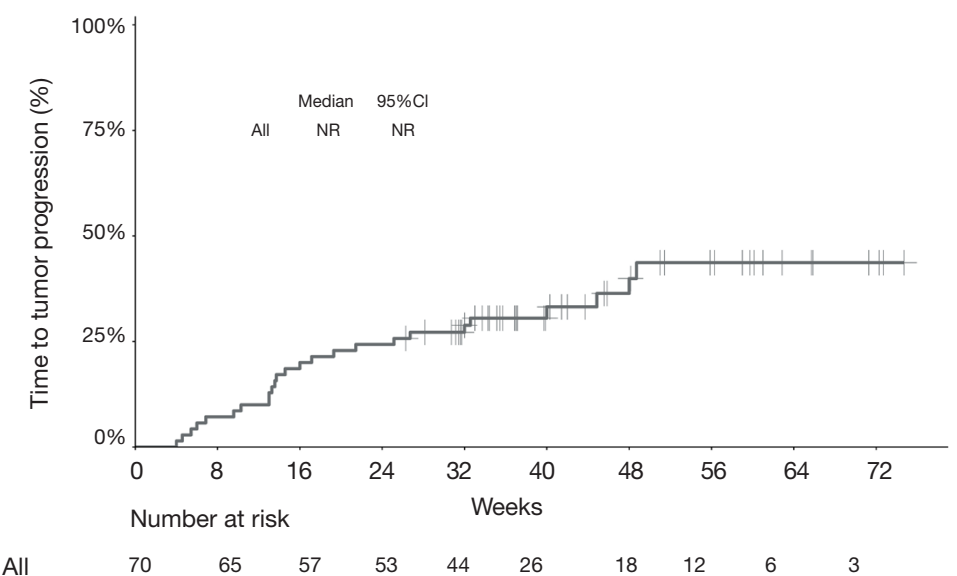

Figure 4 Kaplan-Meier curve showing time to progression for the entire cohort of patients treated with programmed cell death protein-1 (PD-1)-targeted immunotherapy. NR, not reached; CI, confidence interval.

delay due to AEs was needed in 2 patients $(2 / 23,8.7 \%)$ treated with toripalimab, 4 patients $(4 / 33,12.1 \%)$ treated with camrelizumab, and 1 patient $(1 / 14,7.1 \% \%)$ treated with sintilimab. Steroids or immunosuppressive drugs were used to treat an $\mathrm{AE}$ in 10 patients (10/70, 14.3\%), of whom $3(3 / 23,13.0 \%)$ were treated with toripalimab, 6 $(6 / 33,18.2 \%)$ with camrelizumab, and $1(1 / 14,7.1 \%)$ with sintilimab.

\section{Discussion}

At the time of preparing this manuscript, there were no reports available analyzing the efficacy and safety of toripalimab and sintilimab in treating HCC patients. A 


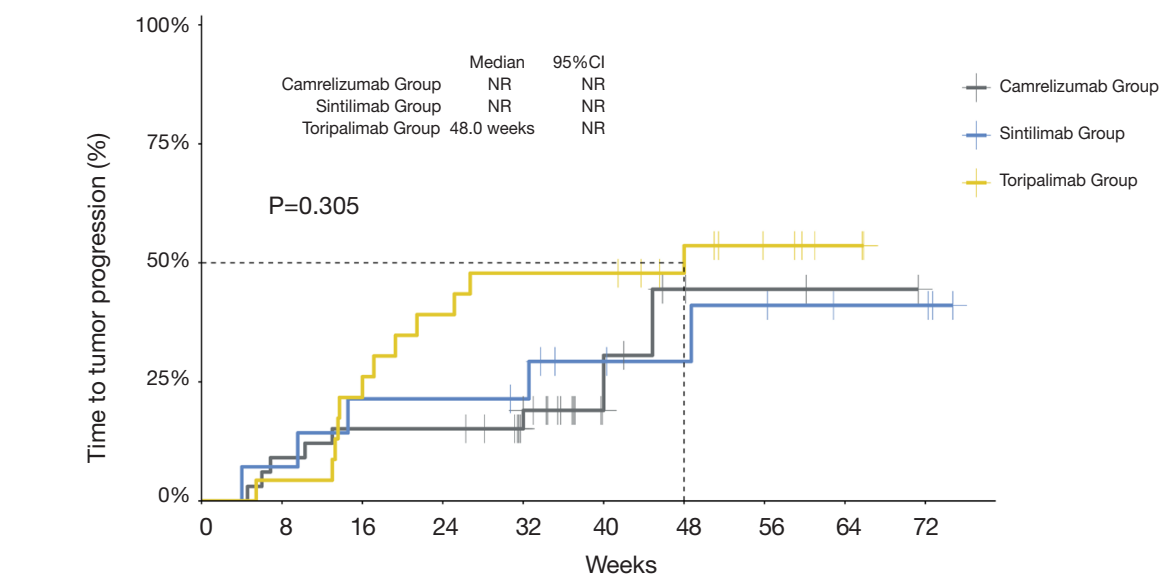

Number at risk

\begin{tabular}{|c|c|c|c|c|c|c|c|c|c|}
\hline Camrelizumab Group & 33 & 30 & 28 & 28 & 22 & 7 & 3 & 2 & 1 \\
\hline Sintilimab Group & 14 & 13 & 11 & 11 & 10 & 7 & 6 & 5 & 3 \\
\hline Toripalimab Group & 23 & 22 & 18 & 14 & 12 & 12 & 9 & 5 & 2 \\
\hline
\end{tabular}

Figure 5 Kaplan-Meier curves showing time to progression for toripalimab-, camrelizumab-, and sintilimab-treated patients. NR, not reached; CI, confidence interval.

Table 3 Treatment related adverse events

\begin{tabular}{|c|c|c|c|c|}
\hline Effect & Toripalimab (n=23) (\%) & Camrelizumab (n=33) (\%) & Sintilimab $(n=14)(\%)$ & All patients $(n=70)(\%)$ \\
\hline Diarrhea & $4(17.4)$ & $5(15.2)$ & $3(21.4)$ & $12(17.1)$ \\
\hline Fatigue & $5(21.7)$ & $4(12.1)$ & $3(21.4)$ & $12(17.1)$ \\
\hline Nausea & $3(13.0)$ & $5(15.2)$ & $3(21.4)$ & $11(15.7)$ \\
\hline Dyspnea/cough & $2(8.7)$ & $4(12.1)$ & $1(7.1)$ & $7(10.0)$ \\
\hline Thyroiditis & $0(0.0)$ & $0(0.0)$ & $4(28.6)$ & $4(5.7)$ \\
\hline Renal & $1(4.3)$ & $1(3.0)$ & $0(0.0)$ & $2(2.9)$ \\
\hline Amylase/lipase increase & $0(0.0)$ & $0(0.0)$ & $0(0.0)$ & $0(0.0)$ \\
\hline Myalgia/myositis & $1(4.3)$ & $4(12.1)$ & $1(7.1)$ & $6(8.6)$ \\
\hline Paresthesia & $2(8.7)$ & $6(18.2)$ & $3(21.4)$ & $11(15.7)$ \\
\hline Hypertension & $6(26.1)$ & $5(15.2)$ & $2(14.3)$ & $13(18.6)$ \\
\hline Dental ulcer & $2(8.7)$ & $4(12.1)$ & $2(14.3)$ & $8(11.4)$ \\
\hline Hoarseness & $1(4.3)$ & $1(3.0)$ & $0(0.0)$ & $2(2.9)$ \\
\hline
\end{tabular}




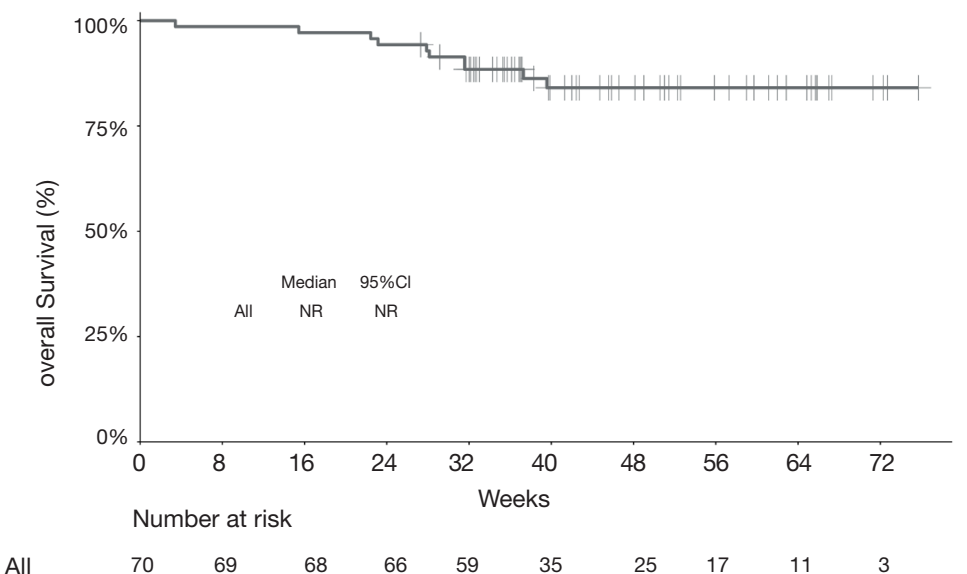

Figure 6 Kaplan-Meier curve showing overall survival for the entire cohort of patients treated with programmed cell death protein-1 (PD-1) -targeted immunotherapy. NR, not reached; CI, confidence interval.

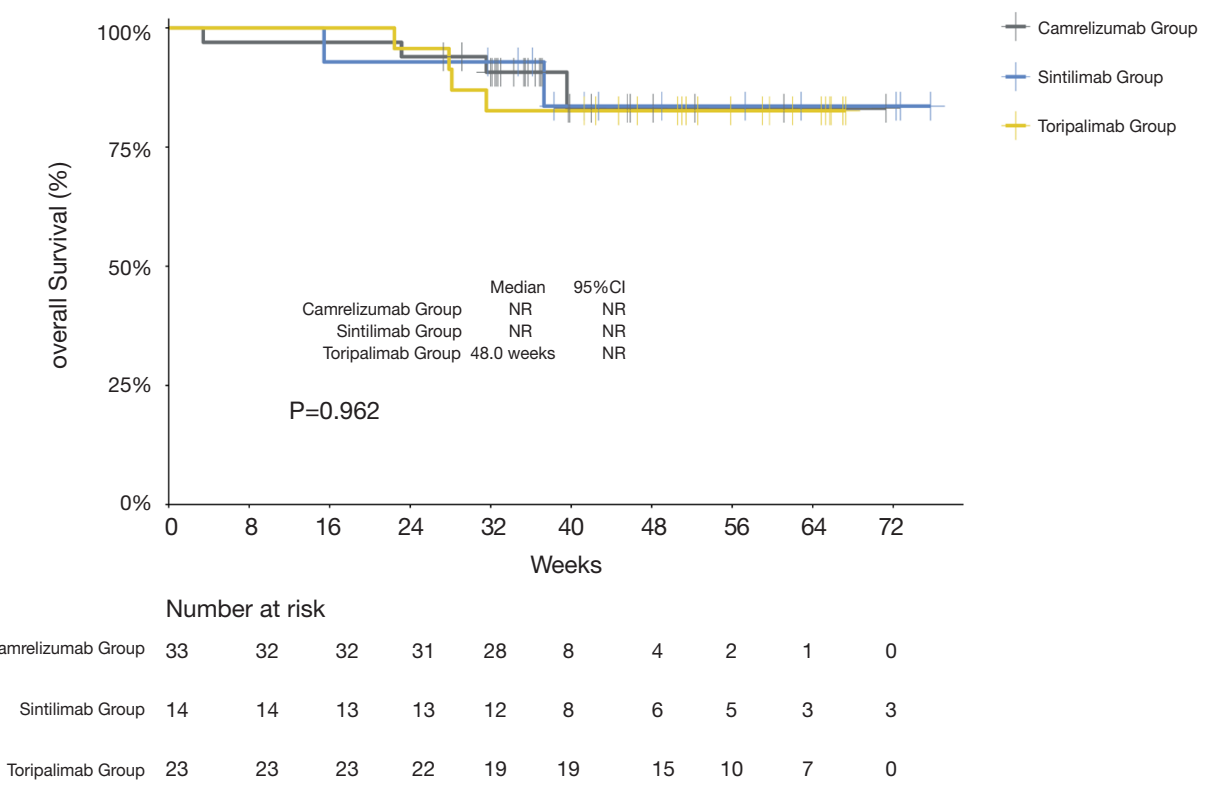

Figure 7 Kaplan-Meier curves showing overall survival for toripalimab-, camrelizumab-, and sintilimab-treated patients. NR, not reached; CI, confidence interval.

phase II/III trial (NCT03794440) of sintilimab combined with an anti-VEGF mAb bevacizumab (IBI305) in HCC is still in its recruitment stage. In a randomized, multicenter phase II/III trial (NCT02989922) of camrelizumab (SHR1210 ) in 217 patients with advanced HCC, the ORR was $13.8 \%$, the 6 -month OS rate was $74.7 \%$, and the TTP and PFS were 2.6 and 2.1 months respectively (28). In this retrospective observational study of $70 \mathrm{HBV}$-associated HCC patients, we have demonstrated the encouraging efficacy of toripalimab, camrelizumab, and sintilimab (ORR 15.4\%, 12.1\%, and 31.3\%, respectively; overall ORR $17.3 \%$ ), in a real-world treatment setting, with welltolerated TRAEs (overall $38.7 \%$ AE rate). Our findings are comparable to the results of the NCT02989922 camrelizumab trial, other PD-1 inhibitor therapy trials (KEYNOTE-224 and CheckMate-040), and real-world observational data in advanced HCC patients (24-26).

This study showed that these PD-1 inhibitor drugs 
were tolerable. Those most common treatment related adverse events were in accordance with the known safety features (27-29). The only one death by $\mathrm{AE}$ is myocarditis in camrelizumab group. This patient did not inform his doctor in the early stage of myocarditis, resulting in delayed treatment, and was in an incurable state. We did not record any reactive capillary hemangiomas in our cohort, which was the most common camrelizumab-related $\mathrm{AE}$ in camrelizumab monotherapy (28). The reason might be that most of the patients were treated in combination with other strategies, especially molecular targeted chemotherapeutics, indicating that combination therapy might reduce the occurrence of some camrelizumab-related AEs or SAEs (35).

Immune evasion through upregulation of the PD-1 pathway is a pivotal mechanism in the progression of HCC. The efficacy of PD-1 checkpoint inhibitor therapy can be affected by the expression of PD-L on tumor cells, the number of different tumor-specific neoantigens that the host immune cells can recognize, and the overall tumor microenvironment (TME) $(18,30)$. Using immunohistochemistry to characterize PD-L1 expression in selected tumor types has been suggested to be associated with the responsiveness of PD-1 treatment. However, the role of such tests has not been established in HCC (36). Higher tumor mutational burden (TMB), results in a broadened display of tumor neoantigens, promoting a polyclonal, efficacious $\mathrm{T}$ cell effector response. $\mathrm{PD}-1$ inhibitors generally have good responses in squamous cell carcinoma, cutaneous melanoma, and lung cancers, whose TMB are above 10 mutations/megabase, with reported ORR ranges from $40 \%$ up to more than $70 \%$ in various clinical trial results (37-39). In contrast, uveal melanoma (UM) is a rare form of ocular tumor, which is believed to have one of the lowest TMBs among all cancers (40). Algazi et al. performed a meta-analysis focusing on UM patients receiving PD-1 inhibitor therapy, and reported only a $3.6 \%$ ORR and a median PFS of as short as 2.6 months (41). HCC typically demonstrates a moderate TMB of roughly 2 mutations/megabase, underscoring the antigenic potential of HCC and perhaps also highlighting one of the reasons for the typical $15-20 \%$ ORR in PD-1 therapy in HCC, which is considerably lower than that of the cancer types with high TMB. In murine models of HCC, dual therapy combining PD-1 blockade with other angiogenesis inhibitor was found to be promising (42). In order to understand and attempt to increase the efficacy of PD-1 efficacy in combined therapy for advanced HCC patients, a number of clinical trials have been set up: NCT03794440, sintilimab with bevacizumab
(VEGF inhibitor); NCT03713593, pembrolizumab plus lenvatinib (VEGR inhibitor); NCT03764293, camrelizumab and the TKI apatinib; and a number of PD-L1 inhibitor trials (NCT03298451, NCT03847428, NCT03434379). Due to its anatomical position, the liver has a critical role in maintaining a state of immune balance and functions as a filter for the blood, clearing potential pathogens while maintaining nonresponsiveness to nonpathogenic antigens. Under normal biological conditions, the presence of a predominantly immunosuppressive microenvironment in the liver might affect disease control in patients with HCC (43).

In China, about $80 \%$ of HCC patients also have HBV infection (31). HBV core antigen has been shown to stimulate IL-10 secretion (32). In HBV-associated HCC patients, the presence of active $\mathrm{HBV}$ infection may further tip the immune balance towards more tolerance in the liver and the whole body via potent immunosuppressive cytokines such as IL-10. Furthermore, viruses possess various strategies for evading the immune system such as modifying host gene expression or deregulating protein function. Several studies have revealed the inability to express of PD-1 and its ligand (PD-L1/B7-H1) on viral antigen specific T-cells and antigen-presenting cells (APCs) respectively (44). Blocking the $\mathrm{PD}-1 / \mathrm{PD}-\mathrm{L} 1$ interaction in vitro was found to relate with exhausted cytokine production and proliferation of these $\mathrm{HBV}$-specific $\mathrm{T}$ cells (45). In our cohort, all 70 patients have active HBV infections; nevertheless, the efficacy of PD-1 therapy is not significantly lower and similar to the published trials and observational studies where both HBV-positive and -negative patients were included. One of the possible causes of the potentially higher than usual ORR is that in our study, $13 / 70(18.6 \%)$ of the patients are staged as BCLC B, while in most of these trials only patients who had progressed on who were intolerant to prior systemic treatments were included. Recently, PD-1 and PD-L1 have been shown to have prominent role in stabilizing antiviral immunity in acute hepatitis B patients with liver inflammation (45). Other research has indicated that postponed upregulation of PD1 and PD-L1 can be ascribed to fulminant hepatitis and subsequently causes acute liver failure $(46,47)$. Moreover, Chen's study in 2016 suggested PD-L1 expression to be positively correlated with the hepatitis B viral load, indicating the blockage of PD-1/ PD-L1 might help to control the chronic inflammatory effect resulting from HBV (48). However, no study has yet analyzed the effect of anti PD-1 drugs in eliminating the 
HBV infection.

Besides the inherent biases in real-world observational studies, there are several limitations to our study. First, the median duration of follow-up was short (44.7 weeks, 95\% CI: 39.9-49.6 weeks). Second, this study was retrospectively designed, although objective endpoints (especially imaging data for tumor responses assessment) were carefully and integrally recorded. Third, 76 of 146 patients were excluded from the final study, which might have reduced the power of statistical analysis. We still believe the above data provide pertinent insights into the subgroups of patients frequently found in our everyday clinical practice and multidisciplinary team (MDT) consultations. Indeed, the strength of our study is that it offers unique real-life data concerning the safety and efficacy of PD-1-targeted immunotherapy with toripalimab, camrelizumab or sintilimab for HBVassociated HCC patients, and, to our knowledge, is first study to evaluate PD-1 blockers in mainland China.

In conclusion, the findings in this study are important for two main reasons: (I) they demonstrate that the efficacy of the three cheaper and domestically developed PD-1 antibodies in treating Chinese HCC patients is similar to that reported in published trials and observational studies; (II) they are novel in that they are derived from the first study to examine the safety and efficacy of these PD-1 inhibitor drugs in a real-world treatment cohort of HBVassociated HCC patients in China. Of course, as nonrandomized retrospective observational data, this study can only provide limited evidence to show that these drugs are efficacious and safe. Without further evidence-based confirmation, these data should not be taken as non-biased or used to inform clinical decision-making. What our study does suggest is that toripalimab, camrelizumab, and sintilimab-the three monoclonal PD-1 checkpoint blockers developed in China-appear to have yielded promising outcomes in advanced HCC patients, with tolerable and manageable side effects that are comparable to the much more expensive, and hence less accessible, alternatives. A multicenter, larger scale, randomized controlled prospective study is needed to further evaluate the efficacy and safety of these drugs with or without other combined treatment modalities.

\section{Acknowledgments}

Funding: This study was partly supported by grants from the Natural Science Foundation of Guangdong Province (No. 2017A030313645), the Self-Financing
Science and Technology Project of Foshan City (No. 2018AB00963), and the WBE Liver Fibrosis Foundation (No. CFHPC2020031). The funding agencies had no role in the study design, data collection and analysis, decision to publish, or preparation of the manuscript.

\section{Footnote}

Reporting Checklist: The authors have completed the STROBE reporting checklist. Available at http://dx.doi. org/10.21037/atm-20-6063

Data Sharing Statement: Available at http://dx.doi. org/10.21037/atm-20-6063

Conflicts of Interest: All authors have completed the ICMJE uniform disclosure form (available at http://dx.doi. org/10.21037/atm-20-6063). The authors have no conflicts of interest to declare.

Ethical Statement: The authors are accountable for all aspects of the work in ensuring that questions related to the accuracy or integrity of any part of the work are appropriately investigated and resolved. Approval for this retrospective study was obtained from the institutional ethics committee (No. NFEC-201903-Y1-01), and written informed consent was obtained from each patient before the procedure began. All procedures performed in this study involving human participants were in accordance with the Declaration of Helsinki (as revised in 2013).

Open Access Statement: This is an Open Access article distributed in accordance with the Creative Commons Attribution-NonCommercial-NoDerivs 4.0 International License (CC BY-NC-ND 4.0), which permits the noncommercial replication and distribution of the article with the strict proviso that no changes or edits are made and the original work is properly cited (including links to both the formal publication through the relevant DOI and the license). See: https://creativecommons.org/licenses/by-nc-nd/4.0/.

\section{References}

1. Parkin DM, Bray F, Ferlay J, et al. Global cancer statistics, 2002. CA Cancer J Clin 2005;55:74-108.

2. Nenu I, Breaban I, Pascalau S, et al. The future is now: beyond first line systemic therapy in hepatocellular carcinoma. Transl Cancer Res 2019;8:S261-74. 
3. Cheung TT. Management of hepatocellular carcinoma: from bench to bedside and beyond. Transl Gastroenterol Hepatol 2019;4:54.

4. Yuen MF, Hou JL, Chutaputti A, et al. Hepatocellular carcinoma in the Asia pacific region. J Gastroenterol Hepatol 2009;24:346-53.

5. Yang JD, Hainaut P, Gores GJ, et al. A global view of hepatocellular carcinoma: trends, risk, prevention and management. Nat Rev Gastroenterol Hepatol 2019;16:589-604.

6. Bae SH, Park HC. Local modalities for inoperable hepatocellular carcinoma: radiofrequency ablation versus stereotactic body radiotherapy. Ann Transl Med 2018;6:S3.

7. Heimbach JK, Kulik LM, Finn RS, et al. AASLD guidelines for the treatment of hepatocellular carcinoma. Hepatology 2018;67:358-80.

8. European Association for the Study of the Liver. EASL Clinical Practice Guidelines: Management of hepatocellular carcinoma. J Hepatol 2018;69:182-236.

9. Vogel A, Cervantes A, Chau I, et al. Hepatocellular carcinoma: ESMO Clinical Practice Guidelines for diagnosis, treatment and follow-up. Ann Oncol 2019;30:871-3.

10. Lin TA, Lin JS, Wagner T, et al. Stereotactic body radiation therapy in primary hepatocellular carcinoma: current status and future directions. J Gastrointest Oncol 2018;9:858-70.

11. Rimassa L, Pressiani T, Merle P. Systemic Treatment Options in Hepatocellular Carcinoma. Liver Cancer 2019;8:427-46.

12. Llovet JM, Ricci S, Mazzaferro V, et al. Sorafenib in advanced hepatocellular carcinoma. N Engl J Med 2008;359:378-90.

13. Kudo M, Finn RS, Qin S, et al. Lenvatinib versus sorafenib in first-line treatment of patients with unresectable hepatocellular carcinoma: a randomised phase 3 noninferiority trial. Lancet 2018;391:1163-73.

14. Bruix J, Qin S, Merle P, et al. Regorafenib for patients with hepatocellular carcinoma who progressed on sorafenib treatment (RESORCE): a randomised, double-blind, placebo-controlled, phase 3 trial. Lancet 2017;389:56-66.

15. Schöffski P, Gordon M, Smith DC, et al. Phase II randomised discontinuation trial of cabozantinib in patients with advanced solid tumours. Eur J Cancer 2017;86:296-304.

16. Smyth MJ, Ngiow SF, Ribas A, et al. Combination cancer immunotherapies tailored to the tumour microenvironment. Nat Rev Clin Oncol 2016;13:143-58.
17. Pardee AD, Butterfield LH. Immunotherapy of hepatocellular carcinoma: Unique challenges and clinical opportunities. Oncoimmunology 2012;1:48-55.

18. Iwai $Y$, Ishida M, Tanaka $Y$, et al. Involvement of PD-L1 on tumor cells in the escape from host immune system and tumor immunotherapy by PD-L1 blockade. Proc Natl Acad Sci U S A 2002;99:12293-7.

19. Thompson RH, Gillett MD, Cheville JC, et al. Costimulatory $\mathrm{B} 7-\mathrm{H} 1$ in renal cell carcinoma patients: Indicator of tumor aggressiveness and potential therapeutic target. Proc Natl Acad Sci U S A 2004;101:17174-9.

20. Blank C, Gajewski TF, Mackensen A. Interaction of PDL1 on tumor cells with PD-1 on tumor-specific T cells as a mechanism of immune evasion: implications for tumor immunotherapy. Cancer Immunol Immunother 2005;54:307-14.

21. Gao Q, Wang XY, Qiu SJ, et al. Overexpression of PDL1 significantly associates with tumor aggressiveness and postoperative recurrence in human hepatocellular carcinoma. Clin Cancer Res 2009;15:971-9.

22. Shi F, Shi M, Zeng Z, et al. PD-1 and PD-L1 upregulation promotes CD8(+) T-cell apoptosis and postoperative recurrence in hepatocellular carcinoma patients. Int J Cancer 2011;128:887-96.

23. Weber J. Immune checkpoint proteins: a new therapeutic paradigm for cancer--preclinical background: CTLA-4 and PD-1 blockade. Semin Oncol 2010;37:430-9.

24. El-Khoueiry AB, Sangro B, Yau T, et al. Nivolumab in patients with advanced hepatocellular carcinoma (CheckMate 040): an open-label, non-comparative, phase 1/2 dose escalation and expansion trial. Lancet 2017;389:2492-502.

25. Zhu AX, Finn RS, Edeline J, et al. Pembrolizumab in patients with advanced hepatocellular carcinoma previously treated with sorafenib (KEYNOTE-224): a non-randomised, open-label phase 2 trial. Lancet Oncol 2018;19:940-52.

26. Scheiner B, Kirstein MM, Hucke F, et al. Programmed cell death protein-1 (PD-1)-targeted immunotherapy in advanced hepatocellular carcinoma: efficacy and safety data from an international multicentre real-world cohort. Aliment Pharmacol Ther 2019;49:1323-33.

27. Keam SJ. Toripalimab: First Global Approval. Drugs 2019;79:573-8.

28. Markham A, Keam SJ. Camrelizumab: First Global Approval. Drugs 2019;79:1355-61.

29. Hoy SM. Sintilimab: First Global Approval. Drugs 2019;79:341-6. 
30. Johnston MP, Khakoo SI. Immunotherapy for hepatocellular carcinoma: Current and future. World J Gastroenterol 2019;25:2977-89.

31. Luo RH, Zhao ZX, Zhou XY, et al. Risk factors for primary liver carcinoma in Chinese population. World J Gastroenterol 2005;11:4431-4.

32. Hyodo N, Nakamura I, Imawari M. Hepatitis B core antigen stimulates interleukin-10 secretion by both $\mathrm{T}$ cells and monocytes from peripheral blood of patients with chronic hepatitis B virus infection. Clin Exp Immunol 2004;135:462-6.

33. National Cancer Institute-Common Toxicity Criteria Adverse Events (CTCAE) Version 4.0. June 14, 2010. Available online: CTCAE_4.03_2010-06-14_ QuickReference_8.5x11.pdf

34. Lencioni R, Llovet JM. Modified RECIST (mRECIST) assessment for hepatocellular carcinoma. Semin Liver Dis 2010;30:52-60.

35. Xu J, Zhang Y, Jia R, et al. Anti-PD-1 Antibody SHR-1210 Combined with Apatinib for Advanced Hepatocellular Carcinoma, Gastric, or Esophagogastric Junction Cancer: An Open-label, Dose Escalation and Expansion Study. Clin Cancer Res 2019;25:515-23.

36. Flynn MJ, Sayed AA, Sharma R, et al. Challenges and Opportunities in the Clinical Development of Immune Checkpoint Inhibitors for Hepatocellular Carcinoma. Hepatology 2019;69:2258-70.

37. Migden MR, Rischin D, Schmults CD, et al. PD-1 Blockade with Cemiplimab in Advanced Cutaneous Squamous-Cell Carcinoma. N Engl J Med 2018;379:341-51.

38. Reck M, Rodríguez-Abreu D, Robinson AG, et al. Pembrolizumab versus Chemotherapy for PD-L1Positive Non-Small-Cell Lung Cancer. N Engl J Med 2016;375:1823-33.

Cite this article as: Chen J, Hu X, Li Q, Dai W, Cheng X, Huang W, Yu W, Chen M, Guo Y, Yuan G. Effectiveness and safety of toripalimab, camrelizumab, and sintilimab in a realworld cohort of hepatitis B virus associated hepatocellular carcinoma patients. Ann Transl Med 2020;8(18):1187. doi: 10.21037/atm-20-6063
39. Robert C, Long GV, Brady B, et al. Nivolumab in previously untreated melanoma without BRAF mutation. N Engl J Med 2015;372:320-30.

40. Yarchoan M, Hopkins A, Jaffee EM. Tumor Mutational Burden and Response Rate to PD-1 Inhibition. N Engl J Med 2017;377:2500-1.

41. Algazi AP, Tsai KK, Shoushtari AN, et al. Clinical outcomes in metastatic uveal melanoma treated with PD-1 and PD-L1 antibodies. Cancer 2016;122:3344-53.

42. Shigeta K, Datta M, Hato T, et al. Dual Programmed Death Receptor-1 and Vascular Endothelial Growth Factor Receptor-2 Blockade Promotes Vascular Normalization and Enhances Antitumor Immune Responses in Hepatocellular Carcinoma. Hepatology 2020;71:1247-61.

43. Jenne CN, Kubes P. Immune surveillance by the liver. Nat Immunol 2013;14:996-1006.

44. Wenjin Z, Chuanhui P, Yunle W, et al. Longitudinal fluctuations in PD1 and PD-L1 expression in association with changes in anti-viral immune response in chronic hepatitis B. BMC Gastroenterol 2012;12:109.

45. Boni C, Fisicaro P, Valdatta C, et al. Characterization of hepatitis $\mathrm{B}$ virus (HBV)-specific T-cell dysfunction in chronic HBV infection. J Virol 2007;81:4215-25.

46. Chen L, Zhang Z, Chen W, et al. B7-H1 up-regulation on myeloid dendritic cells significantly suppresses $\mathrm{T}$ cell immune function in patients with chronic hepatitis B. J Immunol 2007;178:6634-41.

47. Kassel R, Cruise MW, Iezzoni JC, et al. Chronically inflamed livers up-regulate expression of inhibitory B7 family members. Hepatology 2009;50:1625-37.

48. Chen CL, Pan QZ, Zhao JJ, et al. PD-L1 expression as a predictive biomarker for cytokine-induced killer cell immunotherapy in patients with hepatocellular carcinoma. Oncoimmunology 2016;5:e1176653. 